\title{
Generation of haploidy inducers for Cas endonuclease-mediated mutagenesis in barley
}

Satpathy P.*, Mirzakhmedov M., Chamas S., Büchner H., Daghma D.E.S., Kumlehn J. Leibniz Institute of Plant Genetics and Crop Plant Research (IPK) Gatersleben, Germany

* email: satpathy@ipk-gatersleben.de

The production and utilization of doubled haploid (DH) lines is one of the most effective biotechnological measures in modern plant breeding. Each DH line is a unique result of meiotic recombination and yet is itself entirely true-breeding (i.e. homozygous across the entire genome). One way to produce DH lines is to employ haploidy-inducing lines as pollinators. To some extent, the progeny of such crosses include haploid plants that carry only the genome derived from egg cells of the pollinated mother plant. In maize, the capability of inducing haploid progeny proved to be largely due to loss-of-function of the sperm cell-specific PHOSPHOLIPASE A 1 (PLAl) gene. With the aim to produce haploidy-inducing lines for barley, we identified the barley PLAl orthologue that was then subjected to site-directed mutagenesis using Cas endonuclease technology. Among the generated transgenic plants carrying cas 9 and $H v P L A l$-specific guide RNA expression units, nine proved mutated in their target motifs. Selfing of these mutants resulted in haploid progeny with an efficiency of about 5\%. The haploidy-inducing capacity of these mutants was then confirmed and quantified by employing them to pollinate wild-type barley, which resulted in a proportion of 5.8\% haploid progeny. Using such plal knockout lines, we are further about to establish a method of targeted mutagenesis that may be applicable to any barley genotypes of choice. This concept involves the delivery of Cas 9 and target gene-specific guide RNA from sperm cells of cas9/guide RNA-transgenic haploidy inducer lines to the zygote via fertilization, so that the wild-type genomes derived from the maternal parents become accessible to sitedirected mutagenesis, whereas the paternal, transgene-carrying genome is lost during early embryogenesis. Colchicine-induced genome duplication may then give rise to doubled haploid, transgene-free plants, whose exclusively maternally derived genetic makeup is carrying homozygous mutations in their target gene-of-interest. 